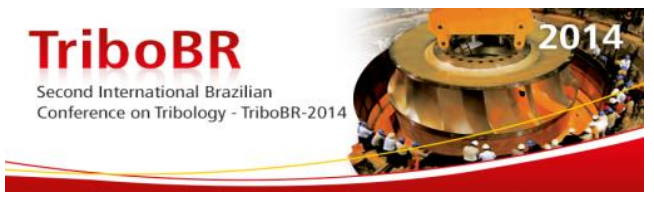

\title{
NUMERICAL MODELING OF ADHESION AND ADHESIVE FAILURE DURING THE UNIDIRECTIONAL CONTACT BETWEEN METALLIC SURFACES*
}

\author{
Eleir Mundim Bortoleto \\ Erika Fernanda Prados ${ }^{2}$ \\ Vanessa Seriacopi ${ }^{3}$ \\ Newton Kiyoshi Fukumasu ${ }^{4}$ \\ Luiz Gustavo Del Bianchi da Silva Lima ${ }^{5}$ \\ Cristiano Fernandes Lagatta ${ }^{6}$ \\ Izabel Fernanda Machado ${ }^{7}$ \\ Roberto Martins Souza ${ }^{8}$
}

\begin{abstract}
A finite element modeling approach was developed to study adhesion phenomena during the unidirectional contact between a two-dimensional plane-strain square and a flat slab. Surfaces were metallic or ceramic, different pairs of materials were analyzed and their adhesion was considered by means of a FORTRAN subroutine (DLOAD), which was connected with commercial finite element code Abaqus and provided surface attractive forces based on the Lennard-Jones interatomic potential. Adhesive loads were considered both during approximation and separation of the surfaces. During the separation step, material transfer between surfaces, due to adhesion, was modeled by means of damage initiation and propagation at the flat slab. The parameters considered in the simulations were normal load, chemical affinity and system size and the different conditions were analyzed by comparison of the interaction forces during approach and withdrawal. This work also presents detailed descriptions of: (i) the evolution of energy dissipation due to adhesion hysteresis, (ii) the formation-growth-breakage of adhesive junctions and (iii) the evolution of stress distribution during the process. Results have indicated that chemical affinity between the bodies in contact is more relevant for adhesion than the applied load. Besides, smaller asperities are less prone to promote adhesive wear, but they may be subjected to high stress concentration effects.
\end{abstract}

Keywords: Adhesion; Adhesive failure; Finite element model; Interatomic potentials.

\footnotetext{
PhD. student, Surface Phenomena Laboratory, University of Sao Paulo, São Paulo, Brazil.

Prof. Dr., Federal University of ABC, Santo André, Brazil.

PhD. student, Surface Phenomena Laboratory, University of Sao Paulo, São Paulo, Brazil.

PhD. student, Surface Phenomena Laboratory, University of Sao Paulo, São Paulo, Brazil.

Msc., Surface Phenomena Laboratory, University of Sao Paulo, São Paulo, Brazil.

Msc., Surface Phenomena Laboratory, University of Sao Paulo, São Paulo, Brazil.

Prof. Dr., Surface Phenomena Laboratory, University of Sao Paulo, São Paulo, Brazil

Prof. Dr., Surface Phenomena Laboratory, University of Sao Paulo, São Paulo, Brazil.
}

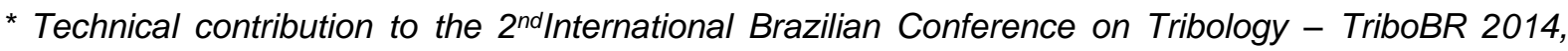
November $3^{\text {rd }}$ to $5^{\text {th }}$, 2014, Foz do Iguaçu, PR, Brazil. 


\section{INTRODUCTION}

The adhesion between two surfaces is generally associated with friction, for example in Bowden \& Tabor's plastic junction theory [1], according to which adhesivelybonded contacting asperities, so called junctions, can form, grow and break during relative sliding [2]. These junctions are formed at points of the real area of contact, due to surface forces of attraction and repulsion that act between the atoms and molecules of two approaching surfaces. The adhesive portion is mainly attributed to London-van der Waals forces, and may be the source of adhesive failure and wear. Therefore, adhesion can promote adverse influence on the performance and durability of many mechanical systems, resulting in economic impacts [3].

According to Hamaker [4], van der Walls interactions between a pair of objects can be obtained by the pairwise summation of the energies acting between all the molecules or atoms in one body with all those in the other body [5]. In that way, pairwise Hamaker approach is a first approximation for van der Waals interactions, which was later complemented by other theories. The Lifshitz theory [6] uses absorption properties of real materials, which considers macroscopic quantities instead of microscopic ones, in order to better estimate Hamaker constants.

The friction force is assumed to be the sum of two contributions: the junctionbreaking forces, related to adhesion, and plastic deformation, related to abrasion. The first ones can be estimated by two major parameters, the junction-breaking shear stress and the junction size, which can be obtained by analytical elastic continuum adhesive contact analyses, such as the JKR (Johnson-Kendall-Roberts [7], DMT (Derjaguin-Muller-Toporov) [8] and MD (Maugis-Dugdale) [9] theories, or by finite element adhesive contact models [10-12]. These analyses [7-12] are also able to predict pull-off force, contact force and contact area [13].

In the JKR model, adhesive forces (attractive tensile forces) are considered only within the contact region, while in the DMT model, adhesive forces are considered only outside of the contact region, with the assumption of Hertzian behavior for the deformed profile [11]. The MD model describes attractive forces based on the Dugdale potential and may be considered the general description of the contact, such that both the JKR and the DMT models are particular cases. Currently, classical contact models such as Hertz, JKR, DMT and Maugis are being used to understand experiments regarding adhesion [14]. However, these classical contact models do not capture adhesive failure and/or material transfer between surfaces, such that comparison with experiments is commonly based only on the loads acting during the contact.

In this work, dry adhesive contact between nanoscale, continuum and homogenous bodies was modeled, considering the case where an indenter applies normal loads on a flat surface. Elastic-plastic finite element analysis was used to capture the physical phenomena that promote material transfer between surfaces, allowing the evaluation of the effect of the system size and material properties on the adhesion forces. Crack generation, which leads to material transfer between surfaces, was estimated, providing information regarding the critical conditions to promote wear due to adhesion.

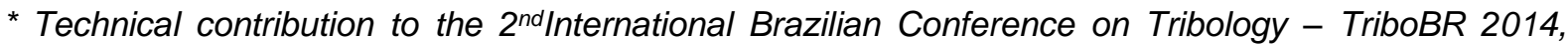
November $3^{\text {rd }}$ to $5^{\text {th }}$, 2014, Foz do Iguaçu, PR, Brazil.
} 


\subsection{Theoretical Considerations}

\subsubsection{Adhesion Forces and Hamaker Expressions}

The Lennard-Jones potential, whose most common form is given by Equation (1), is a simple physical model that approximates the interaction between a pair of neutral atoms or molecules.

$$
w(r)=4 \delta\left[\left(\frac{\sigma}{r}\right)^{12}-\left(\frac{\sigma}{r}\right)^{6}\right]
$$

In Equation (1), $\boldsymbol{w}$ is the Lennard-Jones potential energy function, $\boldsymbol{r}$ is the distance between particles, $\boldsymbol{\delta}$ is the depth of the potential well, and $\boldsymbol{\sigma}$ is the distance at which the inter-particle potential is zero. In this equation, the first term describes the shortrange repulsive interactions and the second, negative term refers to the long-range attractive interactions.

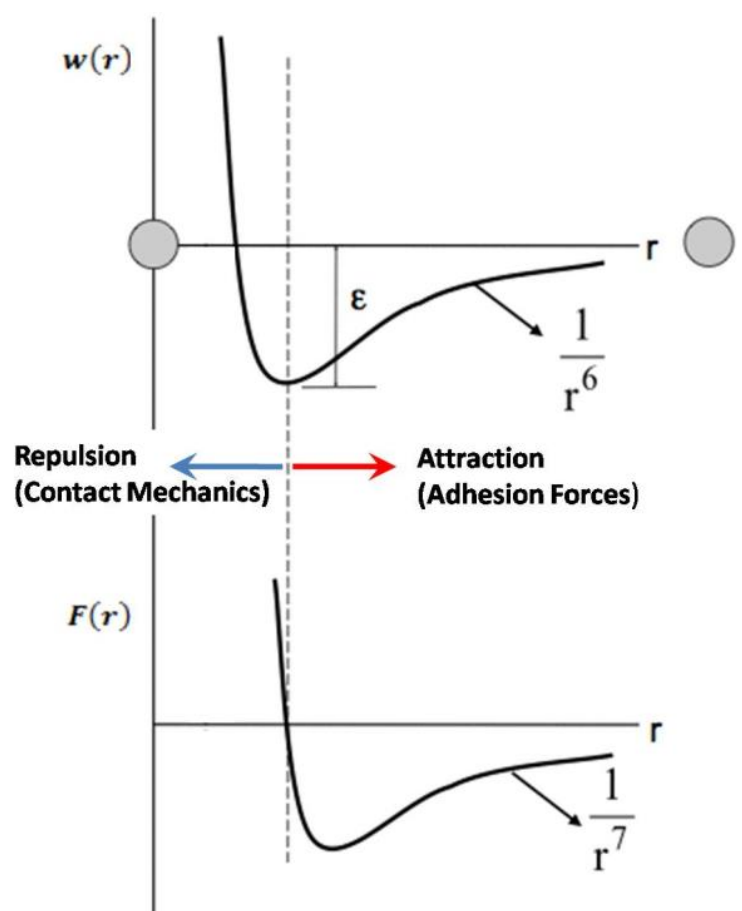

Figure 1.Lennard Jones potential representation and the approach scheme adopted in this work to represent attraction and repulsion forces between surfaces.

According to Equation (1) and Figure 1, when two approaching surfaces or particles are placed closer than a few nanometers, they will be submitted to attraction forces. Thus attraction remains until they touch each other, when repulsion forces may take place. For separation distances below $\sigma$, the two surfaces are in contact and contact mechanics can describe mechanical behavior in this situation. In this situation, the pair potential between two atoms or small molecules can be simplified and be considered purely attractive, for example, considering only van der Walls forces, in which case Equation (1) can be rewritten as:

$$
w(r)=-\frac{C}{r^{6}} \ldots
$$

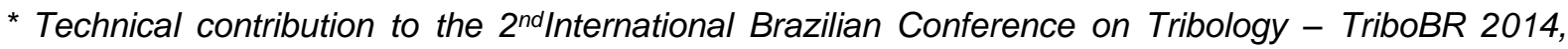
November $3^{\text {rd }}$ to $5^{\text {th }}$, 2014, Foz do Iguaçu, PR, Brazil. 
If the interaction between two flat surfaces (one being infinite) is nonretarded and additive, Equation (2) can be integrated [15] and an interatomic van der Waals pair potential becomes:

$$
w(r)=-\frac{A}{12 \pi r^{2}}(\text { per unit area })
$$

Differentiation of Equation (3) provides adhesion force (per unit area):

$$
F(r)=-\frac{A}{6 \pi r^{3}}(\text { per unit area }) \ldots
$$

where $\boldsymbol{A}$ is the Hamaker constant for the material pair. For two macroscopic phases, 1 and 2, interacting across a medium 3, $\boldsymbol{A}$ can be estimated using McLachlan's equation [15]:

$$
\mathrm{A} \approx \frac{3}{4} k T\left(\frac{\varepsilon_{1}-\varepsilon_{3}}{\varepsilon_{1}+\varepsilon_{3}}\right)\left(\frac{\varepsilon_{2}-\varepsilon_{3}}{\varepsilon_{2}+\varepsilon_{3}}\right)+\frac{3 h \vartheta_{e}}{8 \sqrt{2}} \frac{\left(n_{1}^{2}-n_{3}^{2}\right)\left(n_{2}^{2}-n_{3}^{2}\right)}{\sqrt{\left(n_{1}^{2}+n_{3}^{2}\right)} \sqrt{\left(n_{2}^{2}-n_{3}^{2}\right)}\left\{\sqrt{\left(n_{1}^{2}+n_{3}^{2}\right)}+\sqrt{\left(n_{2}^{2}+n_{3}^{2}\right)}\right\}}
$$

where the input parameters are the dielectric constants, $\boldsymbol{\varepsilon}$, the refractive indices, $\boldsymbol{n}$, and $\boldsymbol{\vartheta}_{\boldsymbol{e}}$ is the absorption frequency.

For two dissimilar materials, the Hamaker constant may be estimated in terms of the geometric mean of the Hamaker constant of each material [16], that is:

$$
A_{12} \approx \sqrt{A_{11} A_{22}}
$$

Or as an alternative [16]:

$$
A_{12}=\frac{2 A_{11} A_{22}}{A_{11}+A_{22}}
$$

\subsubsection{Adhesive Failure and Material Transfer Simulation using XFEM}

Adhesive forces produce stresses and strains that may locally surpass the material strength and lead to material failure and, consequently, to material transfer between surfaces in contact. In this work, the eXtended Finite Element Method (XFEM) was applied to reproduce the material cracks that arise near contact region and promote material transfer and wear. In this technique, a crack is nucleated based on a fracture initiation criterion, which, in this work, was the maximum principal stress [17], and propagates according to a damage evolution criterion based on energy release rate.

\section{MODEL DESCRIPTION}

A finite element analysis was conducted to investigate the contact problem of a linear elastic square punch indenting an elastic-plastic deformable slab. To this end, the elastic adhesive unidirectional contact of a deformable (square or rectangular) asperity with a plane strain surface was modeled using Finite Element solver Abaqus. The analyses were two-dimensional plane strain and were coupled with an ad hoc user FORTRAN subroutine, designed to calculate adhesion forces, which were introduced in the system as forces acting on the surfaces as a function of the separation between them.

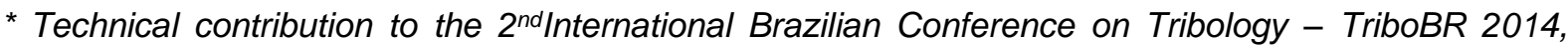
November $3^{\text {rd }}$ to $5^{\text {th }}$, 2014, Foz do Iguaçu, PR, Brazil. 


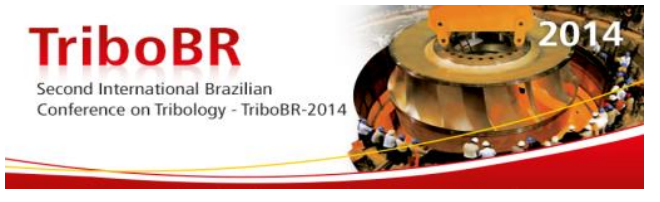

Static implicit modeling and simulation were used to determine stresses, strains and displacement response and also to evaluate material failure using the XFEM capability in Abaqus. The system geometry is presented in Figure 2; which indicates dimensions compatible with the contact of a nanometric asperity indenting over a flat plane surface. Different from some of the analytical adhesion models, such as the DMT model [8], in this work, the interaction between the indenter lateral faces and the slab was considered negligible.

Two different indenter edge lengths were considered in the simulations, as illustrated in Figure 2. Model mesh was composed of 1,092 nodes and 1,000 elements for configuration $\mathrm{A}$, and 982 nodes and 900 elements for configuration $\mathrm{B}$.

In terms of boundary conditions, the slab was fixed at bottom line, and both lateral lines could not move in x-direction. The indenter was allowed to move only in the $y$ direction. The normal load was imposed by means of two penetration depths of the indenter over the slab, $0.1 \mathrm{~nm}$ and $0.2 \mathrm{~nm}$, which will be denoted by 1 and 2 , respectively. This movement was applied to the top line of the indenter. Numerical increment size was restricted to always be lower than $0.01 \mathrm{~nm} / \mathrm{increment}$, in order to avoid abrupt variations in adhesion forces

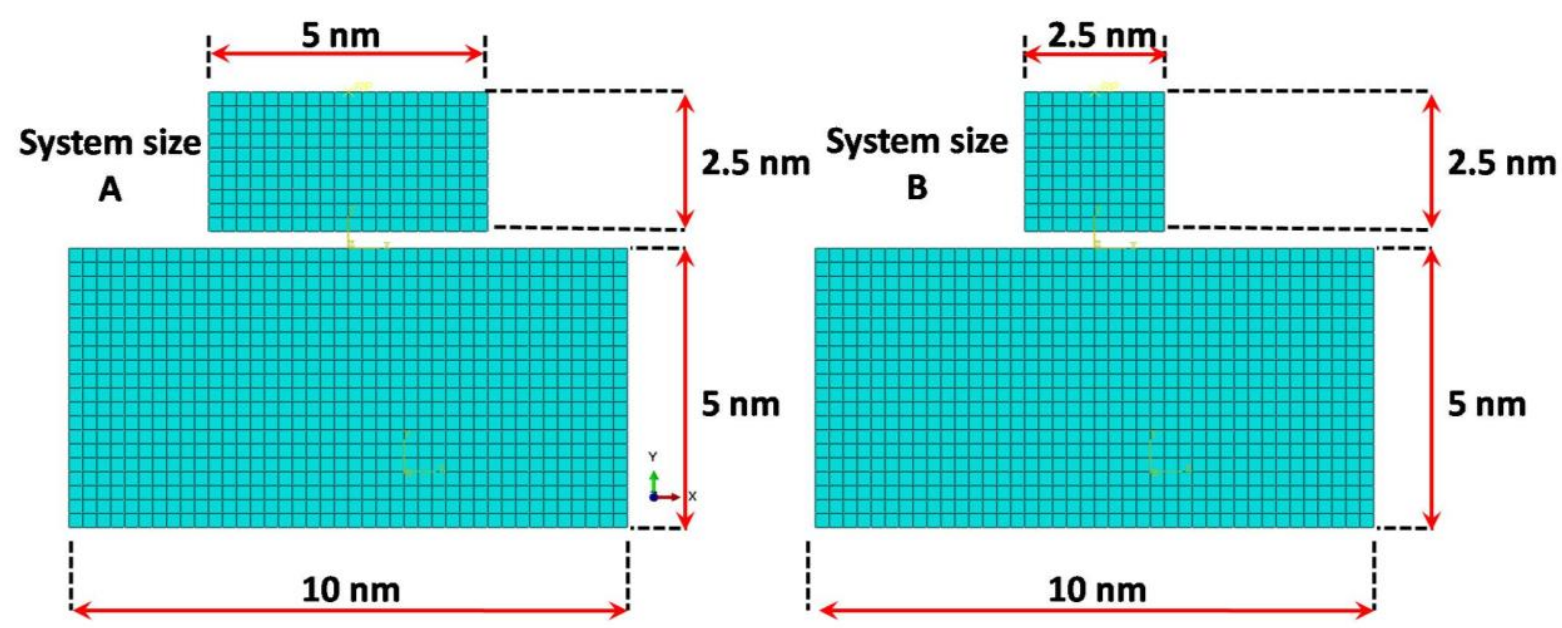

Figure 2.System size and mesh details.

Molecular Dynamics formulation would be more recommended for small size systems simulations. However, a continuum approach at this scale can be expanded to bigger systems in a way that is simpler than atomistic formulations. In order to avoid inconsistencies, element size was chosen to be greater than an atomic diameter. The values for indentation depth and size of contact region in the adhesion contact models were chosen such that bulk and surface were comparable with molecular dynamics simulations.

The most important inputs to the finite element model consist of (i) interfacial geometry; (ii) mechanical properties of sapphire, copper and H13 tool steel; and (iii) adhesion parameters (Hamaker constants). The model inputs are parametrically varied in a systematic way to obtain the variability expected, with 24 combinations, as described in Table 1.

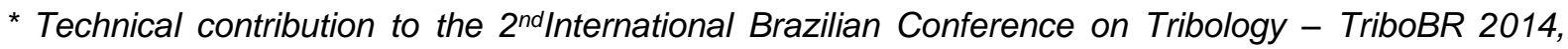
November $3^{\text {rd }}$ to $5^{\text {th }}$, 2014, Foz do Iguaçu, PR, Brazil. 
Table 1.Overview of the studies performed on FEM simulations

\begin{tabular}{|c|c|c|c|c|}
\hline Case & Surface 1 (indenter) & Surface 2 (slab) & Penetration Depth & Indenter Size \\
\hline 01 & Sapphire & H13 (steel) & 1 & A \\
\hline 02 & Sapphire & H13 (steel) & 1 & B \\
\hline 03 & Sapphire & H13 (steel) & 2 & $A$ \\
\hline 04 & Sapphire & H13 (steel) & 2 & $B$ \\
\hline 05 & Sapphire & Copper & 1 & A \\
\hline 06 & Sapphire & Copper & 1 & $B$ \\
\hline 07 & Sapphire & Copper & 2 & $A$ \\
\hline 08 & Sapphire & Copper & 2 & $B$ \\
\hline 09 & H13 (steel) & H13 (steel) & 1 & A \\
\hline 10 & H13 (steel) & H13 (steel) & 1 & B \\
\hline 11 & H13 (steel) & H13 (steel) & 2 & $A$ \\
\hline 12 & H13 (steel) & $\mathrm{H} 13$ (steel) & 2 & $B$ \\
\hline 13 & H13 (steel) & Copper & 1 & A \\
\hline 14 & H13 (steel) & Copper & 1 & B \\
\hline 15 & H13 (steel) & Copper & 2 & $A$ \\
\hline 16 & H13 (steel) & Copper & 2 & $\mathrm{~B}$ \\
\hline 17 & Copper & H13 (steel) & 1 & A \\
\hline 18 & Copper & H13 (steel) & 1 & B \\
\hline 19 & Copper & H13 (steel) & 2 & $A$ \\
\hline 20 & Copper & H13 (steel) & 2 & $\mathrm{~B}$ \\
\hline 21 & Copper & Copper & 1 & $A$ \\
\hline 22 & Copper & Copper & 1 & B \\
\hline 23 & Copper & Copper & 2 & $A$ \\
\hline 24 & Copper & Copper & 2 & $\mathrm{~B}$ \\
\hline
\end{tabular}

Table 2.Mechanical Properties

\begin{tabular}{lccc}
\hline & \multicolumn{3}{c}{ Material } \\
\cline { 2 - 4 } & H13 steel & Cooper & Sapphire \\
\hline Elastic Modulus [GPa] & 210 & 117 & 345 \\
\hline Yield Stress [MPa] & 1650 & 35 & 400 \\
\hline Tensile Strength [MPa] & 1860 & 270 & 415 \\
\hline Poisson's ratio & 0.30 & 0.34 & 0.29 \\
\hline Fracture Toughness [MPa/ $/ \mathrm{m}]$ & 35 & 15 & 3.14 \\
\hline Fracture Energy $\left[\mathrm{J} / \mathrm{m}^{2}\right]$ & 3550 & 1923 & 7.3 \\
\hline Density $\left[\mathrm{kg} / \mathrm{m}^{3}\right]$ & 7800 & 8950 & 3980 \\
\hline
\end{tabular}

The elastic-plastic constitutive properties of the materials were obtained from the existing literature [18-20] and are presented in Table 2. It was imposed that only the elements of the slab could fail and be transferred to the indenter. Thus, no material transfer from the indenter to the slab was allowed.

In this work, one single procedure was adopted in the use of the XFEM method, although it is recognized that further improvements could be possible in the description of copper and other ductile materials [21, 22].

The Hamaker constant, A, (in Joules), represents the strength of van der Waals interactions forces between macroscopic bodies and can be roughly defined as a material property. The values used in the numerical simulations conducted in this work are presented in Table 3 and were obtained in the literature or using Equations (5) and (6). Iron oxide properties were used to define adhesion parameters (Hamaker constant) in the contact of $\mathrm{H} 13$ steel surfaces, since iron and steel surfaces are

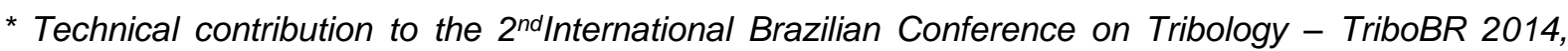
November $3^{\text {rd }}$ to $5^{\text {th }}$, 2014, Foz do Iguaçu, PR, Brazil. 


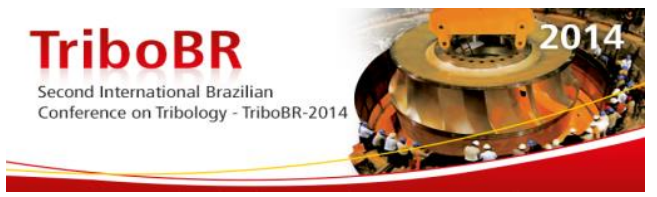

usually found coated with oxide layers; at the same time, mechanical properties of $\mathrm{H} 13$ steel were considered for the material below the surface.

Table 3.Values of Hamaker Constant for some pair materials

\begin{tabular}{cccc}
\hline Surface 1 (indenter) & Surface 2 (slab) & Hamaker constant & \\
\hline Sapphire & Sapphire & $1.56 \mathrm{E}-19$ & ${ }^{* *}$ \\
\hline Sapphire & H13 (steel) & $1.81 \mathrm{E}-19$ & ${ }^{* \star *}$ \\
\hline Sapphire & Copper & $2.50 \mathrm{E}-19$ & ${ }^{* \star *}$ \\
\hline H13 (steel) & H13 (steel) & $2.10 \mathrm{E}-19$ & ${ }^{*}$ \\
\hline Copper & Copper & $4.00 \mathrm{E}-19$ & ${ }^{* *}$ \\
\hline H13 (steel) & Copper & $2.90 \mathrm{E}-19$ & ${ }^{* *}$ \\
\hline${ }^{*}$ Values for iron oxide. Source: . Israelachvili [15] & & \\
${ }^{* * \star}$ Obource: Masliyah and Bhattacharjee [23] & & \\
\end{tabular}

\subsection{Ad hoc Subroutine for Adhesion Forces Calculation}

Based on the potential and force expressions expressed in Equations (3) and (4), a user-defined load FORTRAN subroutine (DLOAD) has been developed, compiled and connected with commercial finite element code Abaqus to calculate adhesion forces during approximation and separation between surfaces.

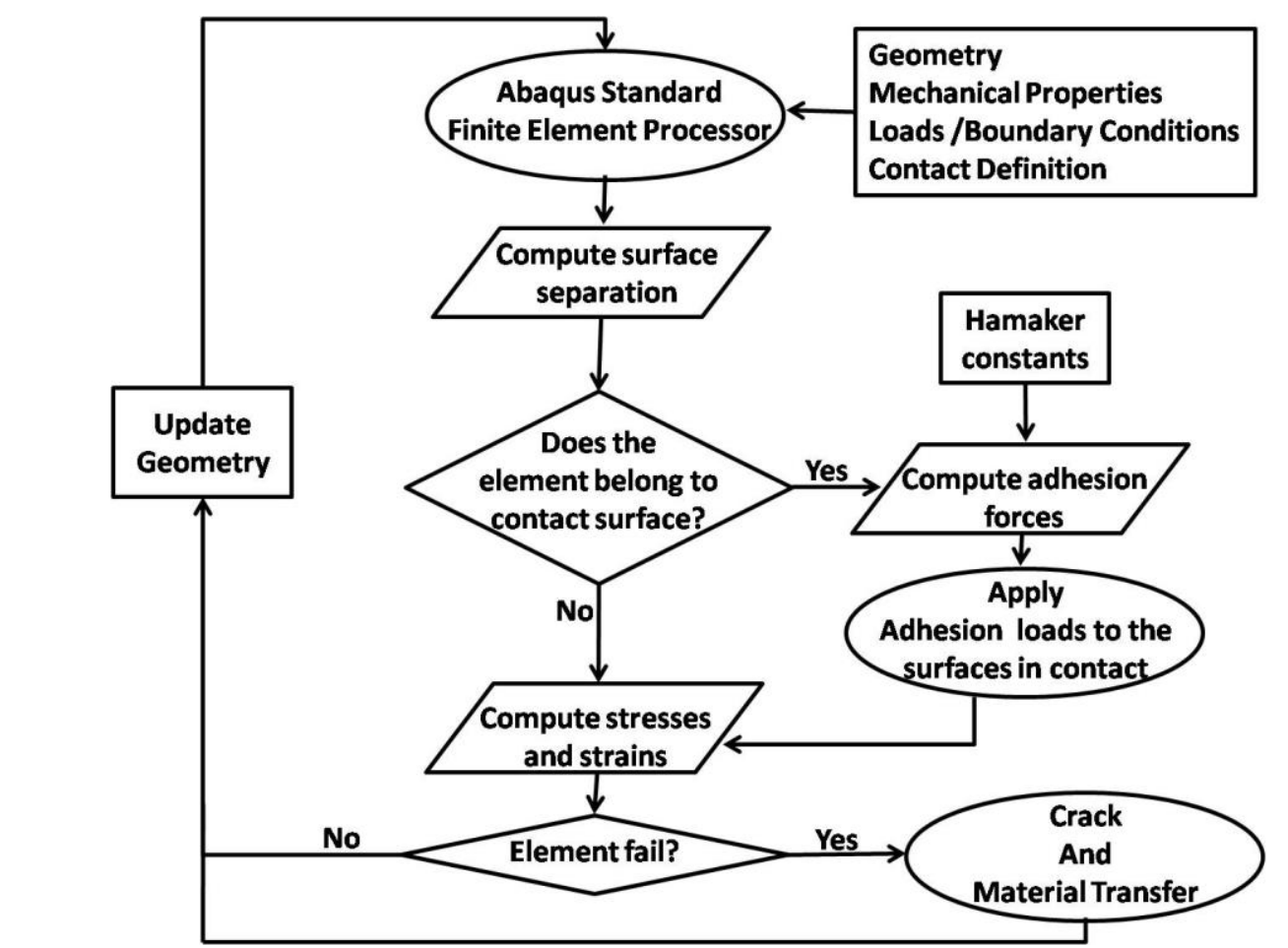

Figure 3.Flowchart for the coupling between FEM software and FORTRAN subroutine DLOAD.

The subroutine iterates for each time increment and for each element searching for the closer element in the opposite surface, updating surface separation for each surface integration point and using this value to feed Equation (4), providing surface force in the elements at each surface. Hamaker constants values presented in Table 3 were used at this stage.

During unloading, the simulation focuses on the adhesive failure. Material transfer between surfaces due to adhesion was modeled by means of damage initiation and propagation criteria by extended finite element method (XFEM), in a way that the

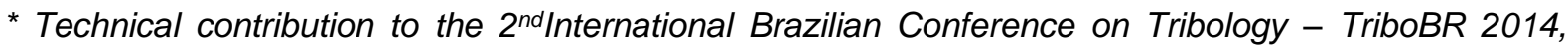
November $3^{\text {rd }}$ to $5^{\text {th }}$, 2014, Foz do Iguaçu, PR, Brazil. 


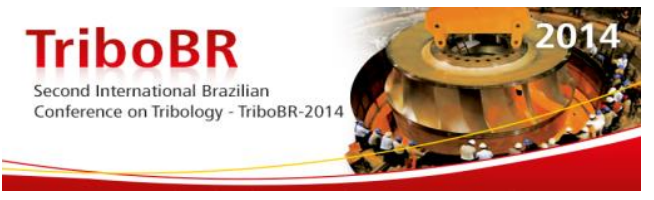

mechanical parameters which have influence on these phenomena could be investigated. Variations of the interaction force during approach and withdrawal, and the dependence of pull-in and pull-off force on the surfaces approximation and separation were calculated.

A simplified flowchart describing the iterative analysis process and the coupling between this subroutine and FEM software is illustrated in Figure 3.

\section{RESULTS AND DISCUSSION}

In this work, the wear related to adhesion phenomena in dry contacts was estimated based on the amount of material transfer from the slab to the indenter. This transfer was evaluated for each of the 24 different combinations of surface pairs and, based on the results that were obtained (Table 4), a wear map for adhesion was proposed.

Table 4. Amount of material transferred between surfaces.

\begin{tabular}{|c|c|c|c|c|c|}
\hline & Material & & Material & & Material \\
\hline Case & Transferred & Case & Transferred & Case & Transferred \\
\hline 01 & 0.00 & 09 & 0.00 & 17 & 0.00 \\
\hline 02 & 0.00 & 10 & 0.00 & 18 & 0.00 \\
\hline 03 & 0.00 & 11 & 0.00 & 19 & 0.00 \\
\hline 04 & 0.00 & 12 & 0.00 & 20 & 0.00 \\
\hline 05 & 1.44 & 13 & 0.41 & 21 & 0.49 \\
\hline 06 & 0.25 & 14 & 0.28 & 22 & 0.30 \\
\hline 07 & 3.25 & 15 & 6.31 & 23 & 3.20 \\
\hline 08 & 1.81 & 16 & 0.89 & 24 & 0.28 \\
\hline
\end{tabular}

The morphology of adhesive failure and energy considerations were also evaluated. The results were organized in different sections, as follows.

\subsection{Types of Adhesive Failure}

Figure 4 shows the four different configurations of adhesive failure and material transfer that were observed as a result of the 24 input combinations. The configuration observed in each case is organized in Table 5.

Table 5.Different pattern configurations observed in adhesive wear results.

Final condition Cases

\begin{tabular}{lc}
\hline No adhesive wear, no cracks & $01,02,03,04,10,11,12,17,18,19$ \\
\hline No adhesive wear, but cracks nucleate & 09,20 \\
\hline Detachment of a thin layer & $06,13,14,16,21,22,24$ \\
\hline Detachment of a thick layer & $05,07,08,15,23$ \\
\hline
\end{tabular}

The amounts of material transferred from one surface to another depend on the slab elastic modulus and other mechanical properties. Besides, materials that have lower hardness resulted in higher material transfer. Increasing values of Hamaker constant as well as increasing penetration depths also contribute to failure and material detachment.

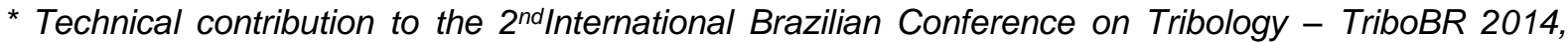
November $3^{\text {rd }}$ to $5^{\text {th }}$, 2014, Foz do Iguaçu, PR, Brazil.
} 


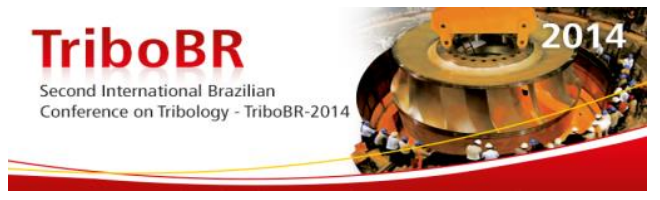

The main reason for the occurrence of detachment of large layers of slab material seems to be the increasing of penetration depth, which in association with increasing contact areas, leads to the highest values of material loss.
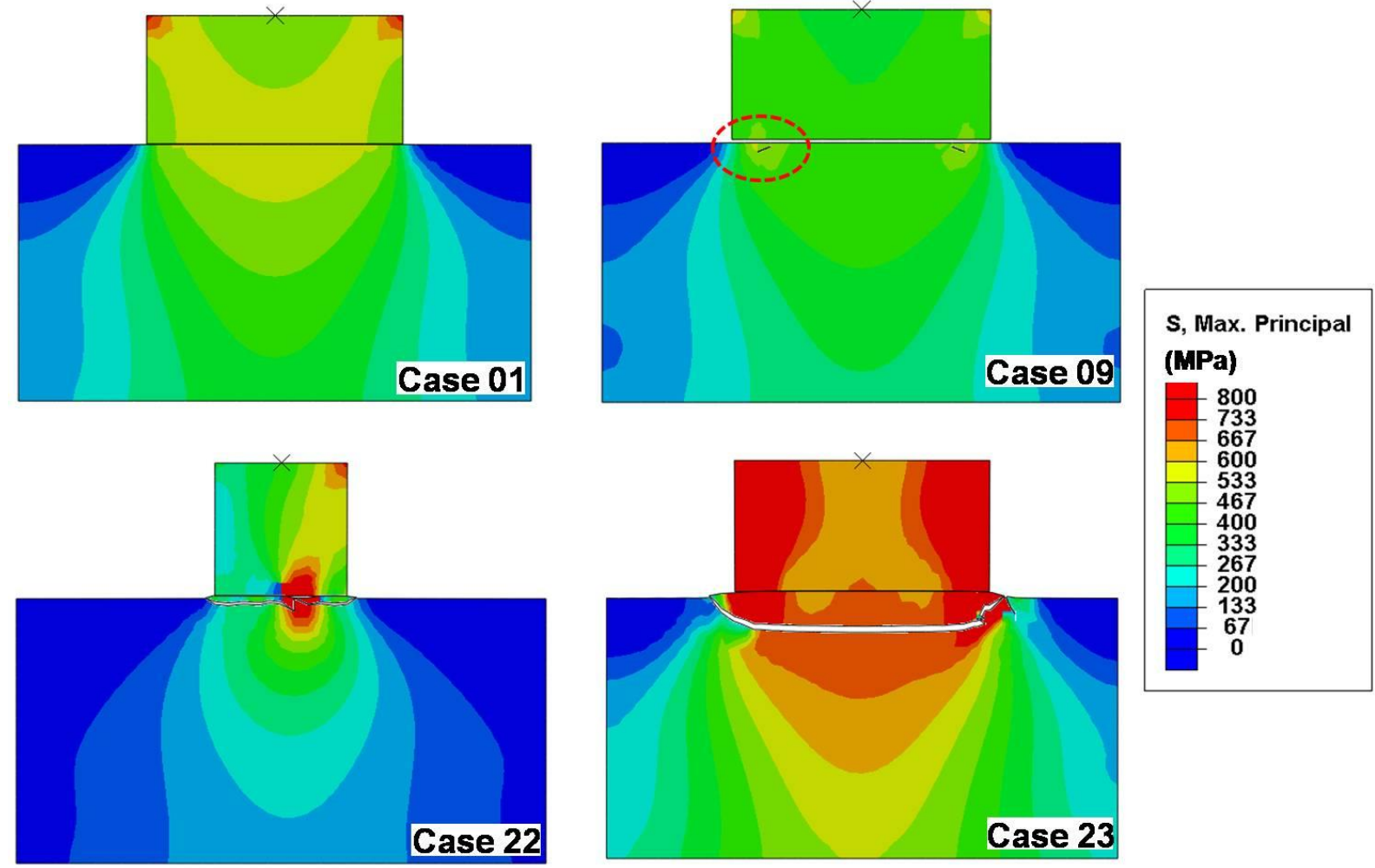

Figure 4.Examples of the 4 Crack types and material transfer in Finite Element Mesh

It is possible to note that for the oxide-covered $\mathrm{H} 13$ slab, a simultaneous increase of load (penetration depth), indenter size and chemical affinity was not able to produce material transfer, which means that, in this condition, higher values of mechanical properties as elastic modulus can minimize the effects of adhesion.

\subsection{Adhesion Hysteresis}

Considering the contact loading and unloading cycle, an adhesion hysteresis may be defined if there is a difference between the forces during the loading and unloading stages. This phenomenon is not captured by classical contact theories, such as Hertz, JKR and Maugis-Dugdale and has been attributed to dynamic effects, such as those due to material viscoelasticity and also to ambient and surface moisture, or attributed to material specific chemical reactions [24]. However, many quasi-estatic contact measurements also display hysteresis and it is possible to have depthdependent hysteresis during perfect elastic contacts.

Figure 5 shows an example of the hysteresis in the behavior of reaction force observed in the numerical results. These results are similar to the ones captured by Molecular Dynamics simulations [24], but using a numerical approach based on continuum mechanics.

It was possible to identify two different behaviors in the hysteresis curves. The first one, related to the simulations that did not result in material transfer between surfaces, shows an unloading force peak slightly greater than the peak related to the loading stage (case 01, for example). On the other hand, in the situations that

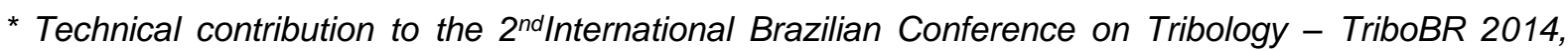
November $3^{\text {rd }}$ to $5^{\text {th }}$, 2014, Foz do Iguaçu, PR, Brazil. 


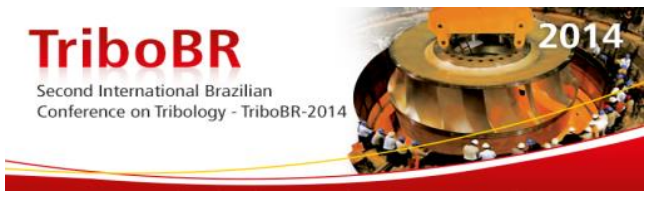

material transfer was observed, for example in case 14, the unloading peak force was less apparent or even absent and always smaller than the loading peak.
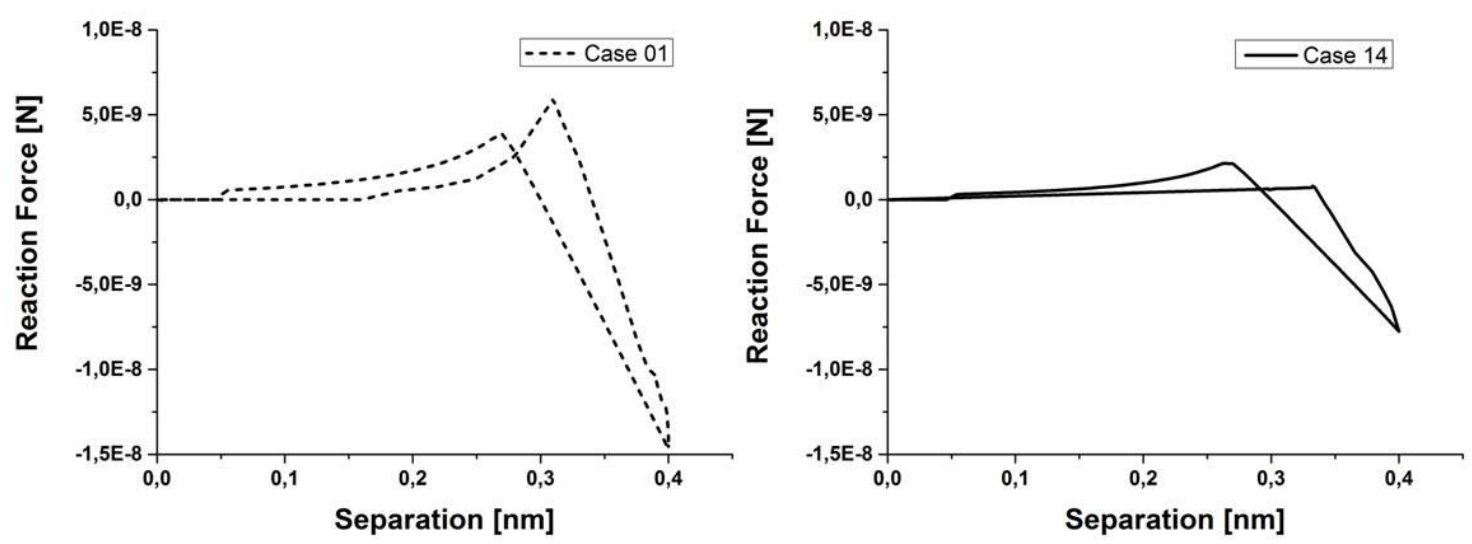

Figure 5. Hysteresis in adhesive contact.

One possible explanation for the observation of the hysteresis phenomenon in this work is that adhesive failure can promote energy loss due to crack propagation in the material, leading to less energy required for separating a pair of bodies in contact. Therefore, an increase in the energy loss due to material failure is equivalent to an adhesive toughening of the contact interface. This observation seems to be in agreement with the work of Zheng et al. [25], in which adhesion hysteresis is built into the assumption that different amounts of work needs to be done when bringing materials into and out off contact.

\subsection{Adhesive Wear and Material Transfer}

Attempts to organize the results obtained in this work led to the definition of a parameter to estimate adhesive wear. This parameter is presented in Equation (7), were $h_{d e p t h}$ is the penetration depth, $L_{\text {real }}$ is the real area of contact, $A_{12}$ is the Hamaker constant, $\mathrm{E}$ is the elastic modulus and $\sigma_{\text {failure }}$ is the material stress strength. This parameter is analogous to Archard's equation [26], since it relates mechanical properties and system characteristics to evaluate wear, but is dedicated to the analysis of adhesion and, for now, restricted to the nanoscale.

$$
\boldsymbol{\alpha}_{\text {adhesion }}=\frac{\mathbf{h}_{\text {depth }} \mathbf{L}_{\text {real }} \mathbf{A}_{12}}{\left(\mathbf{E}_{\text {slab }} / \mathbf{E}_{\text {indenter }}\right) \sigma_{\text {failure }}}
$$

Figure 6 was generated based on this expression, resulting in a proposal for a wearmechanism map for adhesion. Analyzing Figure 6, one can identify 3 different

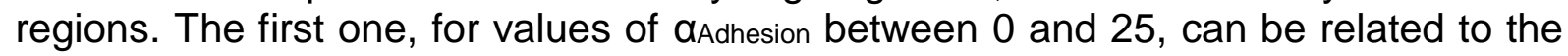
absence of wear due to adhesion between surfaces; values between 25 and 125 can be associated to a mild condition for adhesive wear, while values of aAdhesion greater than 125 indicates a sever condition.

* Technical contribution to the $2^{\text {nd }}$ International Brazilian Conference on Tribology - TriboBR 2014, November $3^{\text {rd }}$ to $5^{\text {th }}$, 2014, Foz do Iguaçu, PR, Brazil. 

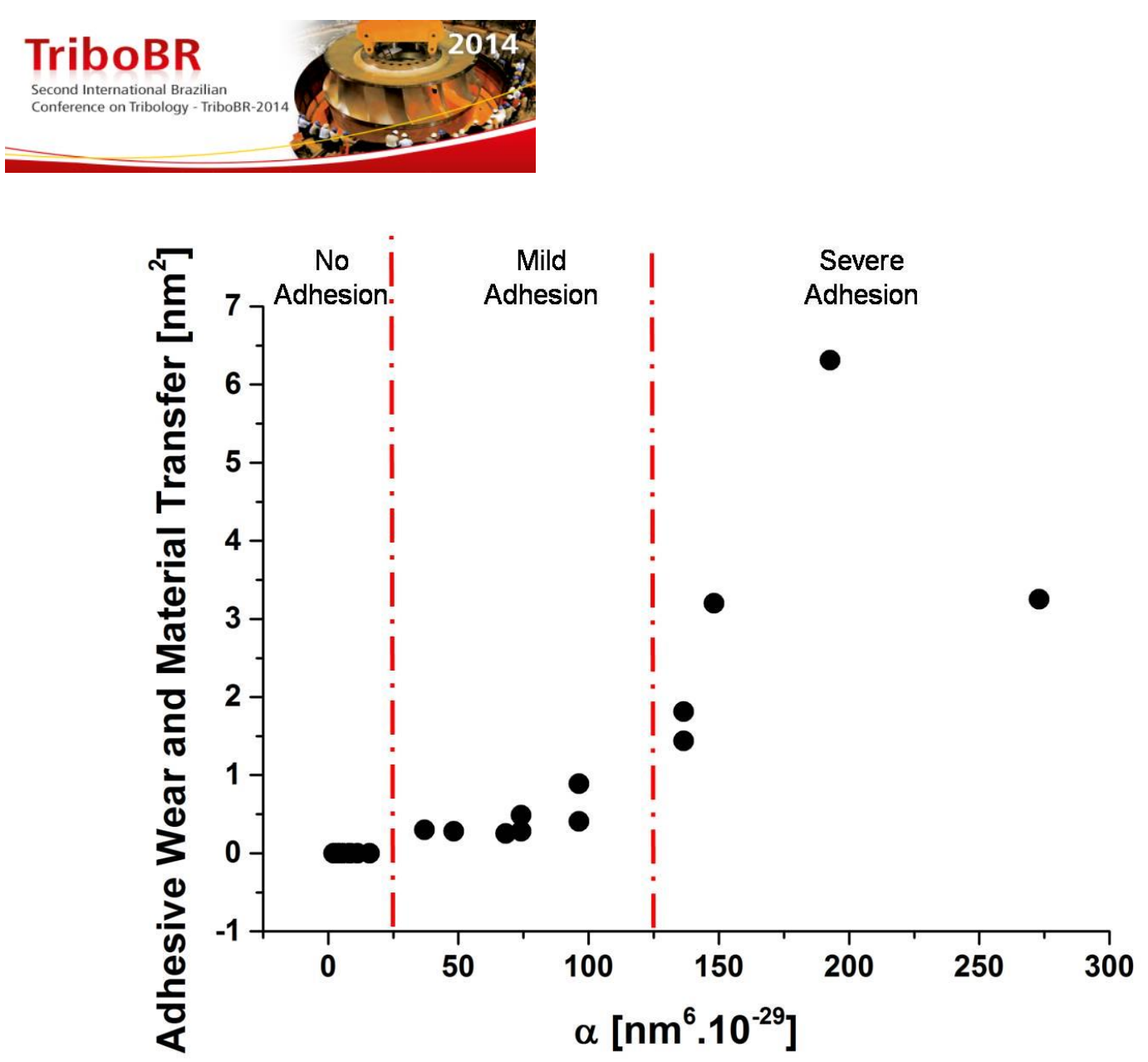

Figure 6.Adhesive Wear computations as a function of a parameter.

\section{CONCLUSION}

The simulations conducted in this work allowed the following conclusions:

- A numerical approach based on continuum mechanics was able to produce results that are coherent with those obtained previously by Molecular Dynamics simulations.

- Adhesion hysteresis could be reproduced with this numerical approach.

- Wear due to adhesion is affected by the chemical affinity, but also by normal load (or indentation depth), contact area and, significantly, by the mechanical properties of the materials in the contact pair.

- There seems to be a strong correlation between the amount of material transferred between surfaces and the proposed parameter $\alpha_{\text {Adhesion. It was }}$ possible to identify 3 different groups of results, which were organized in the form of an adhesive wear map with $\alpha_{\text {Adhesion }}$ as the abscissa.

\section{REFERENCES}

1 Bowden FP, Tabor D. Friction and lubrication of solids. Clarendon Press, Oxford (1964) p. 544.

2 Rabinowicz E. Friction and Wear of Materials, John Wiley \& Sons, New York. 1965; 52108.

3 Buckley DH. Surface effects in adhesion, friction, wear, and lubrication. Tribology Series, 5. Amsterdam 1981.

4 Hamaker HC. The London - van der Waals attraction between spherical particles. Physica 4 (10), (1937) 1058-1072.

5 Berg JC. An introduction to interfaces \& colloids : the bridge to nanoscience. Chemistry \& ChemEng Library (Swain) - Stacks, 2010.

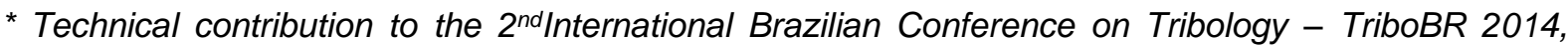
November $3^{\text {rd }}$ to $5^{\text {th }}, 2014$, Foz do Iguaçu, PR, Brazil. 


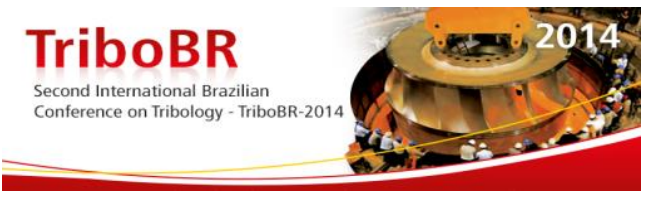

$6 \quad$ Lifshitz EM. Zh. Eksp. Teor. Fiz. 29 94, 1956.

7 Johnson KL, Kendall K. and Roberts AD. Surface energy and the contact of elastic solids, Proc. R. Soc. London A, 1971; 324: 301-313.

8 Derjaguin BV, Muller VM and Toporov YP. Effect of contact deformations on the adhesion of particles, Journal of Colloid and Interface Science, 1975; 53(2): 314-326.

9 Maugis D. Adhesion of spheres: The JKR-DMT transition using a Dugdale model, J. Colloid Interface Sci., 1992; 150: 243-269.

10 Sauer RA, Li S. An atomic interaction-based continuum model for adhesive contact mechanics, Finite Elements in Analysis and Design 43, Issue 5, 2007; ]384-396.

11 Cho SS; Park SH. Finite element modeling of adhesive contact using molecular potential. Tribology International 37 (2004) 763-769.

12 Cho YS, Han H, Kim WD. Numerical Analysis of the Adhesive Forces in Nano-Scale Structure. Journal of Bionic Engineering 3 (2006) 209-116.

13 Cappella B, Dietler G. Force-distance curves by atomic force microscopy. Surface Science Reports 1999; 34:1-104.

14 Prokopovicha P, Pernib S. Comparison of JKR- and DMT-based multi-asperity adhesion model: Theory and experiment. Colloids and Surfaces A, 2011; 383, 95-101.

15 Israelachvili JN. Intermolecular and Surface Forces. Academic Press: London, Third ed. (2011).

16 Sjoblom J. Emulsions and Emulsion Stability. Surfactant Science Series. Marcel Dekker Inc, 1996.

17 Collins JA. Failure of Materials in Mechanical Design - Analysis Prediction Prevention, John Wiley and Sons, 1981.

18 Harvey PD. Engineering Properties of Steels, American Society for Metals, Metals Park, $\mathrm{OH},(1982)$.

19 Iwasa M. and Ueno T. Fracture Toughness of Quartz and Sapphire Single Crystals at Room Temperature. Zairyo, 1981; 30(337): 1001-1004.

20 Wiederhorn SW. Fracture of Sapphire. Journal of the American Ceramic Society, 1969; 52(9): 485-491.

21 Seabra MRR, Sa JMAC Sa, Šuštaric P, Rodic T. Some numerical issues on the use of XFEM for ductile fracture. Computational Mechanics, 2012, Volume 50, Issue 5, pp 611-629

22 Kumar S, Singh IV, Mishra BK. Numerical Investigation of Stable Crack Growth in DuctileMaterials Using XFEM. Procedia Engineering. Vol 64, 2013, Pages 652-660.

23 Masliyah JH, Bhattacharjee S. Electrokinetic and Colloid Transport Phenomena. John Wiley \& Sons, 2006.

24 Kesari H, Doll JC, Pruitt BL, Cai W \& Lew AJ. Role of surface roughness in hysteresis during adhesive elastic contact. Philosophical Magazine Letters. Volume 90, Issue 12, 2010, pages 891-902.

25 Zheng W, Ya-Pu Y. Dhesion elastic contact and hysteresis effect. Chinese Physics, 13:1320-1325, 2004.

26 Archard JF. Contact and rubbing of flat surfaces. Journal of Applied Physics, 24 (1953), pp. 981-988.

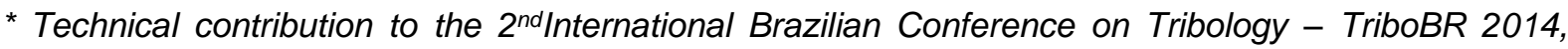
November $3^{\text {rd }}$ to $5^{\text {th }}, 2014$, Foz do Iguaçu, PR, Brazil. 